\title{
Dynamic Models for Addition and Subtraction
}

\author{
Pellumb Kllogjeri ${ }^{1}$, Lindita Kllogjeri ${ }^{2}$ \\ ${ }^{1}$ Department of Mathematics and Informatics, Institute of GeoGebra, University “Aleksander Xhuvani”, Elbasan, \\ Albania \\ ${ }^{2}$ General Secondary School, Labinot-Fushe, Elbasan, Albani \\ Email: pkallogjeri@gmail.com
}

Received 16 August 2015; accepted 2 September 2015; published 7 September 2015

Copyright (C) 2015 by authors and OALib.

This work is licensed under the Creative Commons Attribution International License (CC BY).

http://creativecommons.org/licenses/by/4.0/

(c) $\underset{\mathrm{EY}}{(\mathbf{B})}$ Open Access

\begin{abstract}
One of the most important features of GeoGebra [1] is the coordination of the geometric and algebraic representations, easily observed in GeoGebra window. Using GeoGebra software the teacher can geometrically and fruitfully teach the concepts and algorithms of arithmetic operations in the elementary school. Our paper focuses on two important operations: addition and subtraction in the set of whole numbers. Using GeoGebra features [2], we visually demonstrate the concepts of these two operations and help the students to develop the process of mastering addition and subtraction facts. Our paper aims to achieve two objectives. Firstly, one objective is to teach addition and subtraction using dynamic models that are related to the concept of base ten blocks using 2D model: equal rectangles representing ones, the strips of 10 rectangles representing tens. Secondly, by creating dynamic models for teachers and students we want to: 1) increase teacher pedagogical content knowledge and improve the instructional practice; 2) promote student learning by improving teaching practices and providing capacity-building solutions; 3 ) encourage the teachers to engage themselves in research activity and innovative educational practices and teaching strategies.
\end{abstract}

\section{Keywords}

Place Value, Addition Model, Subtraction Model, Regrouping Algorithm

Subject Areas: Dynamical System, Mathematical Logic and Foundation of Mathematics, Operational Research

\section{Introduction}

In order to make the children get good mathematical progress, the teachers need to lay a secure foundation in

How to cite this paper: Kllogjeri, P. and Kllogjeri, L. (2015) Dynamic Models for Addition and Subtraction. Open Access Library Journal, 2: e1814. http://dx.doi.org/10.4236/oalib.1101814 
mathematical understanding. The key for successful results is to be focused around patterns, relationships and the development of theorems that explain these [3].

The children must be well introduced to the principle of grouping in order that they transfer it as a calculation strategy through their use of the base ten blocks, or of the set representations, or the number line model. The clarity of understanding this principle will assist greatly in their ability to have a complete understanding of the concepts of addition and subtraction. On the other side, the school firmly has the opinion that the concepts need to be taught in the context of "real life" problems so that the children gain full cognitive understanding of the principles of the elementary operations [4]. The designed models related to such operations can easily represent such "real life" problems. The children, taught in such medium, can fully grapple with and gain a full understanding of the principles lying behind the numerical operations. It is recommendable that the teachers use different models for each operation. The more strategies children have access to and the deeper their understanding is, the more effective their calculation processes will be.

The best way to help the children understand the concepts of addition and subtraction is to model and visualize a few place values using base ten blocks. In this model: the place value ten represents 10 ones, the place value hundred represents 10 tens or 100 ones, and the place value thousand represents 10 hundreds, 100 tens or 1000 ones. To build place value concepts, 3D base ten blocks are used: unit cubes representing ones, rod of ten unit cubes representing ten, plate of ten rods representing hundred, and block of ten plates representing thousand. Similarly, in the case of whole numbers less than 1000 we can use 2D models: unit squares representing ones, the strip of 10 unit squares representing 10 and the large square of 10 strips representing 100 . The base ten number system reinforces number concept and applies to addition, subtraction, multiplication and division algorithms.

There are different models for addition and subtraction of whole numbers. Two well-known models for addition are the set model and the number line model. As to subtraction there are four models: the take-away model, the number line model, the missing-addend model, and the comparison model [3]. We will focus on the cases of modelling situations that involve addition and subtraction of whole numbers, using the base ten blocks. The place value concepts help us think about numbers in a flexible way. The place value concepts and the properties of addition and subtraction can be used to find simple sums and differences, and later, of any pair of multi-digit whole numbers. The concept of regrouping is the cornerstone of all algorithms. The strategy used to add multi-digit whole numbers is as follows: add the ones and regroup as necessary to get ten and ones, add the tens and regroup as necessary to get hundred and tens, add the hundreds and regroup as necessary to get thousand and hundreds and so on...

The model for the standard subtraction algorithm builds the minuend and then applies the take-away model of subtraction, subtracting from lower place values to higher place values. As we proceed with the algorithm regrouping occurs as needed. Consider the example: $452-278=$ ?

The procedure, after putting the second number below the first one and place by place from the left to the right:

Step 1 . There are not enough ones to take away 8 ones.

Step 2. Regroup one of the tens as 10 ones, leaving 4 tens. Then 10 ones +2 ones $=12$ ones.

Step 3. There are enough ones to take away 8 ones: 12 ones -8 ones $=4$ ones.

Step 4 . There are not enough tens (are left 4 tens) to take away 7 tens.

Step 5. Regroup one of the hundreds as 10 tens, leaving 3 hundreds. Then 10 tens +4 tens $=14$ tens.

Step 6. There are enough tens to take away 7 tens: 14 tens -7 tens $=7$ tens.

Step 7. There are enough hundreds to take away 2 hundreds: 3 hundreds -2 hundreds $=1$ hundred.

Definitely, $452-278=174$. //

The base ten system can be used by the mathematics educators and teachers to build effective models for addition that allow students to recognize the importance of adding ones to ones, tens to tens, hundreds to hundreds and so on. In the same way, in the case of subtraction: when it is not possible to remove some ones from some ones, or some tens from some tens, or some hundreds from some hundreds etc. then exchange 1 ten for 10 ones and add to some ones, exchange 1 hundred for 10 tens and add to some tens, exchange 1 thousand for 10 hundreds and add to some hundreds etc., if necessary, in order to perform the subtraction of the digits.

It is a strange truth that children can undertake complex calculations well and yet have little understanding of the mathematical processes involved or the numerical principles underpinning them. The reality is that without solid foundations children become vulnerable when new concepts are overlaid on insecure understanding. The 
teacher should be aware that there are students that may use the base ten traditional procedures to model an operation without understanding the respective concepts. The teachers can determine whether their students understand concepts about place value and regrouping, or whether the students are just following mechanically, without fully understanding, by asking them to explain the procedure. That is the main reason to model and visualize a few place values using base ten blocks in order that the students have full understanding of the concepts. We are presenting here the dynamic models built using GeoGebra software [5] that clearly explain the addition and subtraction operations.

\section{The Dynamic Model for Adding Two 2-Digit Numbers}

As a rule, in the primary grades, children learn to add and subtract by using a variety of concrete and pictorial models (counters, number lines, tallies, base ten materials etc.). In higher grades, teachers should focus on learning experiences in which the children continue to use significant models to develop understanding of mental strategies for adding and subtracting multi-digit whole numbers and decimal numbers. Base ten system and its respective materials provide an effective dynamic model for addition that allow children to recognize the importance of adding ones to ones, tens to tens and so on. This model also is used to demonstrate the processes involved in regrouping. The children learn that in the case of having 10 or more ones (10 or more tens etc.), as result of addition operation, is required that 10 ones (10 tens) be grouped to form a ten (a hundred) and the new formed group must be joined with the respective block. The use of such models is so important: they help children understand the procedures involved in addition and subtraction algorithms.

The experience with these models prepares the ground for the students to apply their understanding of computational strategies to determine sums and differences in problems that involve multi-digit whole numbers and decimal numbers [6]. There are students that carry out the procedures mechanically, without thinking about number meaning in the algorithm, because they have little understanding of whether the results in their computations are reasonable. But, if the students develop a variety of models and strategies for adding and subtracting they will move out of their circle of limitations with respect to these two operations. Teachers can help by encouraging students to use a strategy that makes sense to them. Consequently, the students, as learners and doers of the teaching and learning process, will devise strategies that reflect their understanding of the problem, the numbers in the problem, and the operations required to solve the problem. By discussing with the class the various models and strategies used to solve the problem, they can judge the effectiveness of different methods and learn how to adopt them for their own. Learning about various strategies is enhanced when students have opportunities to visualize how the strategies work [5]. This is the main purpose of the two devised dynamic models, using GeoGebra software, related to addition and subtraction. By representing and demonstrating visually the models the students are helped to understand the processes used to add and subtract multi-digit numbers in flexible ways. We will build a dynamic model related to the concept of base ten blocks using 2D model: equal rectangles representing ones, the strip (block) of 10 rectangles representing tens. While is proceeded with the regrouping algorithm occurs as needed. The same model can easily be built to add/subtract two 3-digit numbers, but it takes more time and requires more space in GeoGebra window to build it.

On the other side, the teachers can use the presented procedure to construct models for other cases of addition and subtraction. Furthermore, they can design and develop other procedures using GeoGebra software and the properties of the arithmetic operations. The model of this case and the respective procedure serve as a demonstrative model for the teachers to use in their classroom, equipped with computers, or in computer laboratories. By watching this case the students are able to figure out and build in their minds similar models for other cases of adding/subtracting multi-digit numbers. Manipulatives and visual aids are very important when teaching addition and subtraction.

\subsection{GeoGebra Procedure for the Addition Model: $38+16=$ ?}

1) Open GeoGebra window.

2) Construct 2 sliders. Slider $a$ : $\min =-0.6, \max =0.1$, increment 1 ; Slider $i$ : $\min =0$, $\max =0.25$, increment 0.25 . The selection of the slider values is an option of the teacher depending on the kind of the model s/he will build. They can be fitted in accordance with the size and the position of the blocks and of the rectangles in GeoGebra window. Slider $i$ serves to separate/join the 10 equal rectangles, slider $a$ serves to join the block of 10 grouped equal rectangles with the other blocks on the left. 
3) Assign in GeoGebra window the positions of the blocks (corresponding to tens) and of the rectangles (corresponding to ones) of number 38 (also 16, but below the first), and separate them by a dotted line as in Figure 1. The blocks are on the left side of the vertical axis, the rectangles are on the right side of vertical axis.

4) Construction of rectangles corresponding to ones: using GeoGebra tools, construct 8 equal rectangles of width 0.25 and of height 1 , and in short distance from one another. Clear them from the vertices and from the labels, and assign a colour (green in our case). Construct 6 equal rectangles of the same size, corresponding to the ones of 16, below the 8 first rectangles. All these rectangles are movable, so they can be displaced as needed.

5) Construction of the blocks of 10 rectangles representing tens: construct 3 equal rectangles of width 2.5 and of height 1 (corresponding to tens) which related to 36. Also, construct 1 rectangle of the same size and below the three first rectangles (which relates to 16). Use a different colour for blocks (brown).

6) Divide each of the blocks in 10 equal rectangles as shown in Figure 1. This operation can be performed by the use of the virtual tool "Segment with Given Length" (in our case: 0.25) and dividing the two horizontal sides of each block through successively placing the "Segment with Given Length". After this operation, join the respective points of the opposite sides with segments. There are other ways to divide a segment in equal parts. (Note: to easily join with segment two respective points it is necessary to zoom in the window in order the points be a little far from one another, otherwise you will face difficulties).

7) Construction of the collected blocks: construct 4 equal rectangles of width 2.5 and of height 1 , as above, below the horizontal axis (the red boundary strip). Each of them must be divided in 10 equal rectangles. These are the collection of the given blocks corresponding to tens.

8) Construction of the collected rectangles related to ones: on the right side and below the green rectangles, construct 4 equal rectangles of width 0.25 and of height 1 as in Step 4 . The next 10 rectangles relate to those that will be grouped in one block, therefore we use a different way to construct these rectangles that is described in Step 9.

9) Use the Input Field - construction of the first rectangle. Enter in the Input field the following points: (6.25 + $8 a+i,-0.5)$ and $(6.25+8 a+i$, -1.5$)$. Using "Segment with Given Length" (in our case: 0.25$)$ construct this segment at each of the displayed points and, then construct the rectangle with vertices in these four points. The constructed rectangle is of the same size as the first ones. Clear it from the vertices and from the labels, and assign the same colour (green). Follow the same rule for the construction of the other rectangles by successively entering in the Input Field the following points:

$(6.50+8 a+2 i,-0.5)$ and $(6.50+8 a+2 i,-1.5)$

$(6.75+8 a+3 i,-0.5)$ and $(6.75+8 a+3 i,-1.5)$

$(7.00+8 a+4 i,-0.5)$ and $(7.00+8 a+4 i,-1.5)$

$(7.25+8 a+5 i,-0.5)$ and $(7.25+8 a+5 i,-1.5)$

$(7.50+8 a+6 i,-0.5)$ and $(7.50+8 a+6 i,-1.5)$

$(7.75+8 a+7 i,-0.5)$ and $(7.75+8 a+7 i,-1.5)$

$(8.00+8 a+8 i,-0.5)$ and $(8.00+8 a+8 i,-1.5)$

$(8.25+8 a+9 i,-0.5)$ and $(8.25+8 a+9 i,-1.5)$

$(8.50+8 a+10 i,-0.5)$ and $(8.50+8 a+10 i,-1.5)$

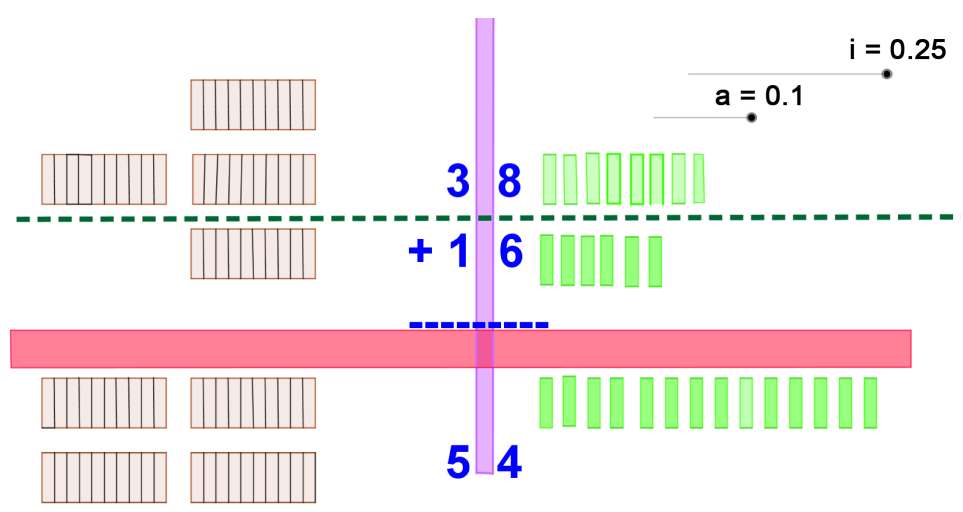

Figure 1. Geometrical representation of the addition operation (Dynamic Model). 
Note 1: In order to construct the rectangles assign to the slider $i$ the value $i=0.25$. Also, use Zoom In tool to increase the distances between the points.

The construction of the model is completed.

\subsection{Displaying the Equation of the Addition in GeoGebra Window}

Show on GeoGebra window the following text:

1. Using the virtual tool "ABC-Text" register the numbers 38 and 16 (representing the addends) and their sum: 54.

2. Arrange the text in order they form the equation in vertical form, also the dotted line as border line of addends and their sum, by selecting the place, their size and their colour as desired.

\subsection{Demonstration}

Click on slider $i$ and assign for it the value $i=0$, then the 10 rectangles will be grouped in one block. Click on slider $a$, and slide its point to the left. Then the formed block will jump to the left joining the 4 blocks on the left side. The new situation of the 5 blocks and 4 rectangles resembles the sum of the given numbers (54). The simulation of the above model is a resemblance of the mental process for adding two 2-digit numbers.

Note 2: The respective applet is shared on GeoGebra tube:

You can download the free application from the GeoGebra Web site- - http://www.geogebra.org and play with it by visiting https://tube.geogebra.org/student/m1118849.

\section{The Dynamic Model for Subtracting Two 2-Digit Numbers}

\subsection{GeoGebra Procedure for the Subtraction Model: $43-16=$ ?}

1) Open GeoGebra window.

2) Construct 2 sliders. Slider $a$ : $\min =0$, $\max =0.5$, increment 0.5 ; Slider $i$ : $\min =0$, $\max =0.25$, increment 0.25 . The selection of the slider values is an option of the teacher depending on the kind of the model s/he will build. They can be fitted in accordance with the size and the position of the blocks and of the rectangles in GeoGebra window. Slider $i$ serves to separate/join the 10 equal rectangles of the particular block, slider $a$ serves to transfer the block of 10 grouped equal rectangles to the left side of the rectangles.

3) Assign in GeoGebra window the positions of the blocks (corresponding to tens) and of the rectangles (corresponding to ones) of number 43 (also 16, but below the first), and separate them by a dotted line as in Figure 2. The blocks are on the left side of the vertical strip (violet colour), the rectangles are on the right side of vertical strip.

4) Construction of rectangles corresponding to ones: using GeoGebra tools, construct 3 equal rectangles of width 0.25 and of height 1 , in short distance from one another and far from the vertical strip. Clear them from the vertices and from the labels, and assign a colour (green in our case). Construct 6 equal rectangles of the same size, corresponding to the ones of 16 , below the 3 first rectangles. All these rectangles are movable, so they can be displaced as needed.

5) Construction of the blocks of 10 rectangles representing tens: construct 4 equal rectangles of width 2.5 and of height 1 (corresponding to tens) which relate to 43. Also, construct 1 rectangle of the same size and below the 4 first rectangles (which relates to 16). Use a different colour for blocks (brown).

6) Divide each of the blocks in 10 equal rectangles as in the case of dynamic model for the addition.

7) Construction of the blocks related to the difference: construct 2 equal rectangles of width 2.5 and of height 1 , as above, below the horizontal axis (the red boundary strip). Each of them must be divided in 10 equal rectangles. These are the left blocks as result of the subtracting process.

8) Construction of the left rectangles as result of the subtracting process which relate to ones: on the right side and below the green rectangles, construct 7 equal rectangles of width 0.25 and of height 1 as in Step 4 .

9) Use the Input Field - construction of the ten rectangles that can be separated/joined in a particular block. Enter in the Input field the same points (10 points) as in the case of the dynamic addition model starting with the point: $(6.25+8 a+i, 3)$ and $(6.25+8 a+i$, 4). Construct the respective rectangle, clear it from the vertices and from the labels, and assign the same colour (brown). Follow the same rule for the construction of the other rectangles by successively entering in the Input Field the following points: 


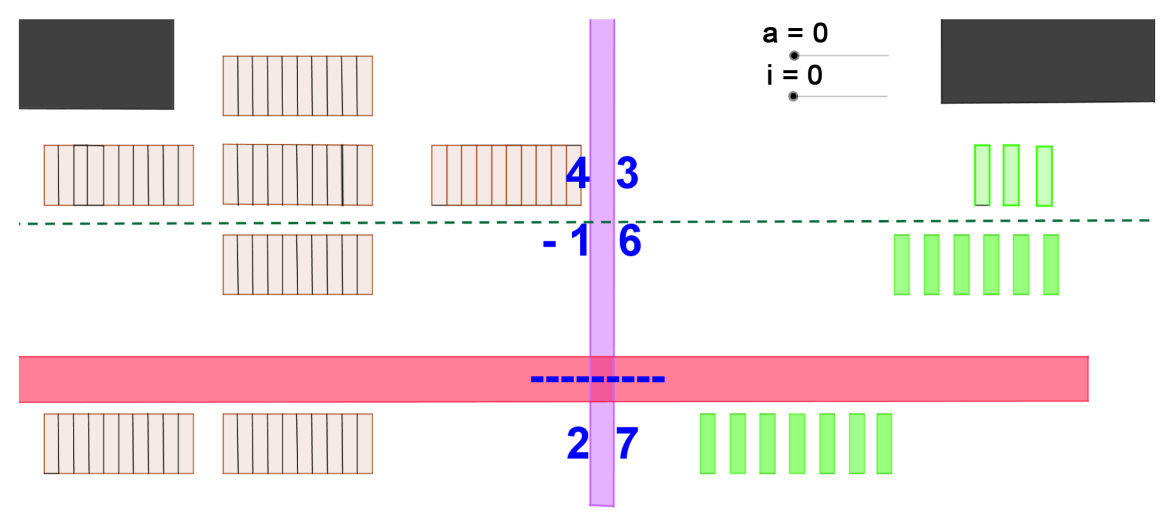

Figure 2. Geometrical representation of the subtraction operation (Dynamic Model).

$(6.50+8 a+2 i, 3)$ and $(6.50+8 a+2 i, 4)$

$(6.75+8 a+3 i, 3)$ and $(6.75+8 a+3 i, 4)$

$(7.00+8 a+4 i, 3)$ and $(7.00+8 a+4 i, 4)$

$(7.25+8 a+5 i, 3)$ and $(7.25+8 a+5 i, 4)$

$(7.50+8 a+6 i, 3)$ and $(7.50+8 a+6 i, 4)$

$(7.75+8 a+7 i, 3)$ and $(7.75+8 a+7 i, 4)$

$(8.00+8 a+8 i, 3)$ and $(8.00+8 a+8 i, 4)$

$(8.25+8 a+9 i, 3)$ and $(8.25+8 a+9 i, 4)$

$(8.50+8 a+10 i, 3)$ and $(8.50+8 a+10 i, 4)$

\subsection{Displaying the Equation of the Subtraction in GeoGebra Window}

1) Use the "ABC-Text" tool to register variable $t$ as follows: open "Text" and, on the open window, click at Objects. On the displayed window of Objects choose the letter $t$ (representing tens). If you register variable $n$ in any other way (e.g. by typing $n$ ) then it is not possible that $t$ take different values.

2) Enter in the Input field the function $t=4-2 a$ in order to display it on Algebra window.

3) Use again the "ABC-Text" tool to register letter o (representing ones) in the same way: open "Text" and on the open window click at Objects. On the displayed window of Objects choose the letter $o$.

4) Enter in the Input field the function $o=3+40 i$ in order to display it on Algebra window.

5) Arrange the texts in order they form the equation of the division by selecting the place, their size and their colour as desired.

6) Arrange the text in order they form the equation in vertical form, also the dotted line as border line between the two numbers and their difference, by selecting the place, their size and their colour as desired.

\subsection{Demonstration}

Click on slider $a$ and assign for it the value $a=0.5$, then one of the blocks, representing tens, will jump from left to the right side (the side of ones). Click on slider $i$, and assign for it the value $i=0.25$. Then the transfer block will decompose in 10 equal rectangles. The new situation of 3 blocks and 13 rectangles allows us to find the difference between the two sets of blocks and the difference between the two sets of rectangles. The result is 2 blocks and 7 rectangles which represent 2 tens and 7 ones, respectively. There are two black curtains (represented by black rectangles) on GeoGebra window which are used to cover the blocks and the rectangles that are taken away during the subtracting process. The simulation of the above model is a resemblance of the mental process for subtracting two 2-diget numbers.

Note 2: The respective applet is shared on GeoGebra tube:

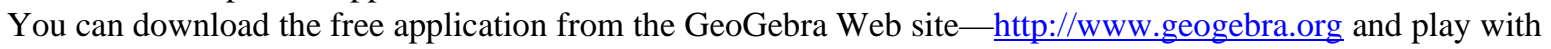
it by visiting http:/tube.geogebra.org/material/show/id/1118887.

\section{Conclusions}

By representing and demonstrating visually the models, the students are helped to understand the processes used 
to add and subtract multi-digit numbers in flexible ways. The use of dynamic models is very important: they help children understand the procedures involved in arithmetic elementary algorithms.

Our purpose is to conduct and utilize applied research in special areas of educational content, instruction and technology to improve student learning, enhance teaching and learning, and meet the needs of diverse learners. Working alongside with the other researchers and GeoGebra specialists, the main objectives are:

- Help the teachers to use technology effectively in their teaching process to improve student learning;

- Promote student learning by improving teaching practices and providing capacity-building solutions;

- Encourage the teachers to engage themselves in research activity and innovative educational practices and teaching strategies;

- Encourage the teachers to use designed models, develop other models and share their works and results within GeoGebra community and with other education communities [7]. The teachers can use the presented procedures to construct models for other cases of addition and subtraction. Furthermore, they can design and develop other procedures using GeoGebra software and the properties of the arithmetic operations;

- Contribute in the professional development programs.

Note 1. Any teacher can learn and be equipped mostly on his/her own by experimentation, adding here the help from suggestions on GeoGebra Wiki, Twitter, and Googling stuff from the online help. Our paper is a challenge for all the teachers of the elementary school to use such dynamic models, explore and test the effectiveness of the proposed methods in their teaching process, and online publicize their results. We welcome remarks, new suggestions and are open for cooperation.

Note 2. The way that the two simple concepts are treated with maybe a complex one in content, but the visual side is not a problem for the children: it helps them to construct mental models. As mentioned above, our purpose is to help teachers to use technology effectively in their teaching process and encourage the teachers to use the designed models and develop other models. The provided procedures are very difficult to understand for children, but they are not area of children learning or participation.

\section{References}

[1] GeoGebra (2009). http://www.geogebra.org

[2] Hohenwarter, J. and Hohenwarter, M. (2008) Introduction to GeoGebra. 3-20.

[3] Ministry of Education (2006) Number Sense and Numeration, Grades 4 to 6, Vol. 1-6. Queens Printer for Ontario, Toronto.

[4] Division of Higher Education (2002) Information and Communication Technology in Education (A Curriculum for Schools and Programme of Teacher Development). UNESCO, France, 8-22.

[5] Hohenwarter, M. and Preiner, J. (2007) Dynamic Mathematics with GeoGebra. The Journal of Online Mathematics and Its Applications, 7, Article ID: 1448.

[6] Fierro, R.D. (2013) Mathematics for Elementary School Teachers. Charlie van Wagner, USA, 69-118.

[7] Hohenwarter, M. and Lavicza, Z. (2009) The Strength of the Community: How GeoGebra Can Inspire Technology Integration in Mathematics Teaching. Mathematics, Statistics, Operation Research Connections, 9, 3-5. http://dx.doi.org/10.11120/msor.2009.09020003 